\title{
Hidden Markov Modeling of Human Pathological Gait using Laser Range Finder for an Assisted Living Intelligent Robotic Walker
}

\author{
Xanthi S. Papageorgiou, Georgia Chalvatzaki, Costas S. Tzafestas and Petros Maragos
}

\begin{abstract}
The precise analysis of a patient's or an elderly person's walking pattern is very important for an effective intelligent active mobility assistance robot. This walking pattern can be described by a cyclic motion, which can be modeled using the consecutive gait phases. In this paper, we present a completely non-invasive framework for analyzing and recognizing a pathological human walking gait pattern. Our framework utilizes a laser range finder sensor to detect and track the human legs, and an appropriately synthesized Hidden Markov Model (HMM) for state estimation, and recognition of the gait patterns. We demonstrate the applicability of this setup using real data, collected from an ensemble of different elderly persons with a number of pathologies. The results presented in this paper demonstrate that the proposed human data analysis scheme has the potential to provide the necessary methodological (modeling, inference, and learning) framework for a cognitive behavior-based robot control system. More specifically, the proposed framework has the potential to be used for the classification of specific walking pathologies, which is needed for the development of a context-aware robot mobility assistant.
\end{abstract}

\section{INTRODUCTION}

Mobility problems, particularly concerning the elderly population, constitute a major and ever growing issue in our society. Walking disabilities are common and impede many activities of daily living and have strong impact on productive life, independence, physical exercise, and self-esteem, [1], [2]. Current demographics show that the elderly population (aged over 65) in industrialized countries is increasing constantly, [3]. Having these issues addressed will have great socioeconomic effects. Robotics seem to fit naturally to the role of assistance, since it can incorporate features such as posture support and stability, walking assistance, navigation in indoor and outdoor environments, health monitoring etc.

The motivation in this work is to use intelligent mobile robotic mechanisms (e.g. a rollator, Fig. 1) which can monitor and understand specific forms of human walking activity in their workspace [4], in order to deduce their needs regarding mobility and ambulation, and to provide contextbased support, [5] and intuitive assistance in domestic environments.

In this paper we address the challenge of developing a reliable pathological walking recognition system utilizing a laser sensor that detects and tracks the user (which does not interfere with human motion), based on a Hidden Markov

This research work was supported by the European Union under the project "MOBOT" with grant FP7-ICT-2011-9 2.1 - 600796.

The authors are with the School of Electrical and Computer Engineering, National Technical University of Athens, Greece, xpapag@mail.ntua.gr, gchal@central.ntua.gr, ktzaf, maragos@cs.ntua.gr

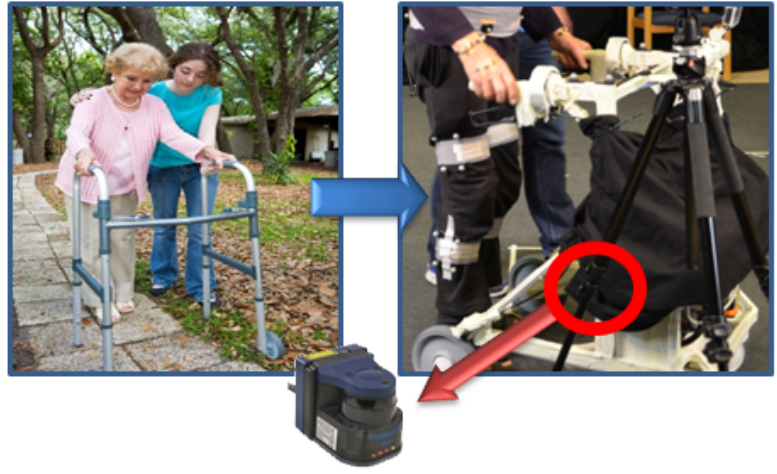

Fig. 1: Right: Typical passive assistive device for elderly. Left: A robotic platform based on the first rollator prototype equipped with a Hokuyo Laser Sensor aiming to record the gait cycle data of the user (below knee level).

Model (HMM) for gait modeling and classification.

The automatic classification and modeling of specific physical activities of human beings is very useful for many technical and biomechanical applications. A number of research groups worldwide, are actively pursuing research, currently investigating problems related to the development of smart walking support devices, aiming to assist motorimpaired persons and elderly in standing, walking and other mobility activities, as well as to detect abnormalities and to assess rehabilitation procedures [6]-[10].

For the extraction of gait motions, different types of sensors have been used, from gyroscopes and accelometers to cameras, etc., [11]-[15]. Other approaches refer to human detection and tracking, or recognition of human activity utilizing laser sensors, and in some cases complementary with cameras, or force sensors (e.g. [16]). Towards this direction, modeling human locomotion by estimating the legs' kinematic parameters with respect to the mobility aid is essential. The detection and tracking of humans is a common problem. Most research work focuses on detecting and tracking human legs from static sensors, as in pedestrian tracking (e.g. [17]), or from laser scanners mounted on mobile robotic platforms for person following [18], [19]. Approaches for tracking users of robotic walkers can be found in [20]-[22], while [23] refers to a walker that detects and tracks Parkinson's patients.

Time series data can be modelled by Hidden Markov Models (HMMs). In HMMs only the output of the model is visible to the observer and the states of the model (corresponding to a physical event) are not observable, in other words are hidden, [24]. The versatility of HMMs makes 
them useful in extracting human patterns. Apart from their prominent application in speech recognition, [25], HMMs are also used in a number of pattern recognition applications, gesture recognition, [26], human activity analysis, [27] and biometric gait recognition, [28]-[30].

This paper proposes a flexible and readily extensible approach for gait representation and characterisation based on early results presented in [31]. In this work, an HMM is employed to model the pathological gait and to analyse transitions between specific gait phases. As opposed to most of the literature available on the topic, the gait analysis approach presented in this paper is completely noninvasive based on the use of a typical non-wearable device. Instead of using complex models and motion tracking approaches that require expensive or bulky sensors and recording devices that interrupt human motion, the observation data used in this work are provided by a standard laser rangefinder sensor mounted on a robotic rollator platform. There were many challenges in designing a robust system for the detection and tracking of patients, that have not been confronted in literature, like the difficulty of the collected data from real patients, the deformability of the detected legs shape due to clothing and the difficulty of constructing a reliable linear motion model to describe the variability of pathological gait. The objective of this work is to design a framework so that it can be possible to actively incorporate many different gait patterns as a subsystem within a larger cognitive behaviourbased context-aware robot control framework (that embodies several walking morphologies, including turning and maneuvering motions). Furthermore, this framework has the potential to be used for the classification of various walking pathologies and related impairments, and for actively and cognitively being augmented with new patients with mobility difficulties.

\section{HUMAN GAIT CYCLE ANALYSIS}

The human gait motion analysis and modeling is based on the basic requisite of the act of walking which is the periodic movement of each foot from one position of support to the next. This element is necessary for any form of bipedal walking to occur, no matter how distorted the pattern may be by an underlying pathology, [32]. This periodic leg movement is the essence of the cyclic nature of human gait.

Traditionally the gait cycle has been divided into eight events or periods, but these are sufficiently general to be applied to any type of gait, five during stance phase (the foot is on the ground) and three during swing (the same foot is no longer in contact with the ground), [33]. The stance phase events (1-5) and the swing phase events (5-8) are as follows, Fig. 2, [33], [34] (as a percentage of the total duration of the gait cycle): 1. Initial contact $(0 \%)$ - [IC] Heel strike initiates the gait cycle and represents the point at which the bodys centre of gravity is at its lowest position. 2. Loading response $(0-10 \%)$ - [LR] - Foot-flat is the time when the plantar surface of the foot touches the ground. 3. Midstance (10-30\%) - [MS] - Midstance occurs when the swinging (contralateral) foot passes the stance foot and the

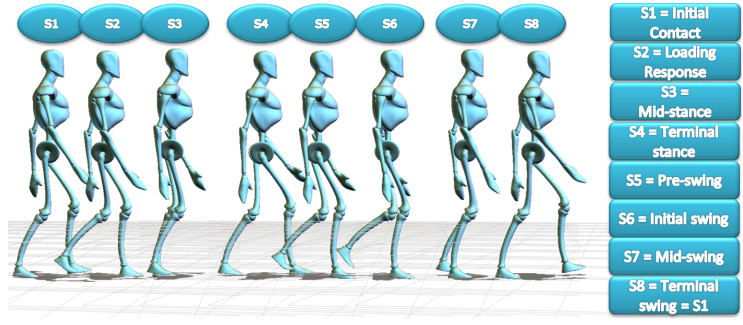

Fig. 2: Internal phases of human normal gait cycle.

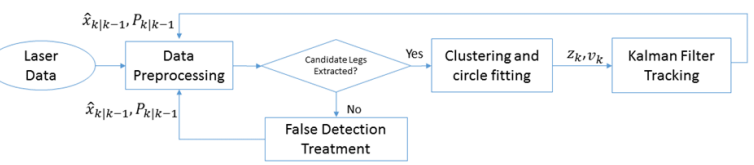

Fig. 3: Overview of the system of detection and tracking of the user's legs.

body's centre of gravity is at its highest position. 4. Terminal stance $(30-50 \%)$ - [TS] - Heel-off occurs as the heel loses contact with the ground and push off is initiated via the triceps muscles, which plantar flex the ankle. 5. Preswing $(50-60 \%)$ - [PW] - Toe-off terminates the stance phase as the foot leaves the ground. 6. Initial Swing (60-70\%) [IW] - Acceleration begins as soon as the foot leaves the ground and the subject activates the hip flexor muscles to accelerate the leg forward. 7. Midswing (70-85\%) - [MW] -Midswing occurs when the foot passes directly beneath the body, coincidental with midstance for the other foot. 8. Terminal swing $(85-100 \%)$ - [TW] - Deceleration describes the action of the muscles as they slow the leg and stabilize the foot in preparation for the next heel gait cycle.

In this paper we have used the seven gait phases of walking cycle in order to analyze the gait cycle, since the TW phase is characterized by heel strike that is an equivalent trigger to the IC phase, and therefore those phases are treated as identical.

\section{RECOGNITION OF GAIT CYCLE PHASES BASED ON HIDDEN MARKOV MODEL}

Hidden Markov Models are well suited for gait recognition because of their statistical properties and their ability to reflect the temporal state-transition nature of gait. They can provide temporal segmentation of time sequence of observations by estimating an optimal state sequence. This optimal state sequence is found using the Viterbi decoding algorithm.

The seven gait phases can define the hidden states of the HMM, Fig. 2. As observables, we utilize several quantities that represent the motion of the subjects' legs,(relative position w.r.t. the laser, velocities, etc.), which are estimated using sequential signals from a laser sensor installed on a robotic rollator that follows the subject's motion. The state and observations at time $t$ are denoted as $s_{t}$ and $O_{t}$, respectively. The seven states at time $t=1,2, \ldots, T$, where $T$ is the total time, are expressed by the value of the (hidden) variable $s_{t}=i$, for $i=1, \ldots, 7$, where $1 \equiv I C / T W$, $2 \equiv L R, 3 \equiv M S, 4 \equiv T S, 5 \equiv P W, 6 \equiv I W$, and $7 \equiv M W$. 
The observations at time $t$, are represented by the vector $O_{t}=\left[o_{t}^{1} \ldots o_{t}^{k}\right]^{T}$, for $k=1, \ldots, 9$, where $o_{t}^{1} \equiv x^{R}, o_{t}^{2} \equiv y^{R}$, $o_{t}^{3} \equiv x^{L}, o_{t}^{4} \equiv y^{L}, o_{t}^{5} \equiv v_{x}^{R}, o_{t}^{6} \equiv v_{y}^{R}, o_{t}^{7} \equiv v_{x}^{L}, o_{t}^{8} \equiv v_{y}^{L}$, and $o_{t}^{9} \equiv$ Dlegs. These signals are the coordinates and the velocities along the axis for right and left leg, and Dlegs is the distance between legs. The observation data (derived from the raw laser sensor data) are modeled using a mixture of Gaussian distributions.

In normal gait cycle the gait phases follow each other sequentially, while in pathological walking some of the gait phases may disappear. Thus, we have assumed that the proposed HMM is a left-to-right model.

\section{GAIT FEATURE EXTRACTION: DETECTION AND TRACKING}

The gait features (composed of dynamic parameters, such as the positions of the legs with respect to the rollator, their velocities and their interdistance), which are essential to characterize gait cycle properties as analysed in [31], and to signal transitions between gait phases (as the observation features fed in the HMM), are extracted from the laser sensor data at each time instant. The necessary walking parameters for the HMM analysis are estimated by the detection and tracking of the user's lower limbs. Our approach is a recursive system with a substantial forward-backward interaction between the detection and tracking of the user.

\section{A. Data Preprocessing and legs extraction}

Data Preprocessing is triggered in each time frame (Fig. 3 ), during which we define a rectangle area in the scanning plane, a search window, where we expect the user to be with respect to the rollator. The initial search window is predetermined and wide enough, while in the subsequent frames it is adjusted. We use a simple background extraction method based on thresholding criteria. The laser points that are not detected inside the search window are discarded, while the remaining are separated into groups, corresponding to detected objects according to the Euclidean distance between consecutive laser points. In cases of discontinuities of laser points, due to fluctuations of the device, or due to the object's deformable surface (common in creasing pants), instead of having one laser group describing an object, we end up with more. In such cases the adjacent laser groups are merged according to an euclidean distance threshold. Finally, any laser group that contains less than a specific number of points is deleted. The remaining laser groups formulate the candidate legs. The candidate legs extraction is successful, if we end up with two candidates, corresponding to the legs. The treatment of cases of less or more laser groups is described bellow.

\section{B. Clustering and Circle Fitting}

Clustering and Circle Fitting are fed with the candidate legs, Fig. 3. Those enter a K-means++ clustering algorithm, [35], to classify the left and right leg. Instead of using the highly noisy centroid-mean of each cluster given by Kmeans, we take as consensus that the human limbs can be represented as cylinders, and therefore can be seen as circles in the scanning plane. We use nonlinear least squares circle fitting on the leg clusters, with a constant pre-computed radius, in order to approach the actual planar leg centers. In that way we have a compact representation of the legs, which is reducing the influence of the shape deformations of the laser groups. The detected legs' centers compose the observation vector, $z_{k}$, for the tracking process.

\section{Kalman Filter Tracking}

The tracking of the user's legs is performed by a Kalman Filter (KF) algorithm, in which the observation vector consists of the detected leg centers, Fig. 3. The state vector we used has eight parameters: $x_{k}=\left[\begin{array}{llllllll}x^{L} & y^{L} & x^{R} & y^{R} & v_{x}^{L} & v_{y}^{L} & v_{x}^{R} & v_{y}^{R}\end{array}\right]^{T}$, where $\left(x^{L}, y^{L}\right)$ and $\left(x^{R}, y^{R}\right)$ are the cartesian coordinates of the centroid legs' positions w.r.t laser, and $\left(v_{x}^{L}, v_{y}^{L}\right),\left(v_{x}^{R}, v_{y}^{R}\right)$ are their velocities along the axes. We model the dynamic of the legs as dynamic points, with the acceleration being the control. Since we have no direct influence on the acceleration (it is "user" generated) nor any measurements, we treat the acceleration as a process noise. In the current implementation we model the acceleration as having the same statistics throughout the gait. We are already working on a more elaborate model, where the estimated gait phase is fed back to alter the statistics of the acceleration.

We use the standard KF state equations: $x_{k}=A_{k} x_{k-1}+$ $B_{k} u_{k-1}+w_{k}$, where $\mathrm{k}$ refers to time, $x_{k}$ is the state vector, $u_{k}$ is the input vector, $A_{k}$ is the state transition matrix, $B_{k}$ is the input matrix and $w_{k}$ is the process noise with normal probability distribution $p\left(w_{k}\right) \sim N\left(0, Q_{k}\right)$, where $Q_{k}$ is the process noise covariance matrix. The observation vector $z_{k}$ of the true state is updated according to the standard equation: $z_{k}=H_{k} x_{k}+v_{k}$, where $H_{k}$ is the observation matrix which maps the true state space into the observed space, and $v_{k}$ is the observation noise. The state transition matrix $A_{k}$, the input matrix $B_{k}$, as well as the observation matrix $H_{k}$, have the following form, respectively:

$A_{k}=\left[\begin{array}{cc}I_{4} & A_{1} \\ \emptyset_{4} & I_{4}\end{array}\right], \quad B_{k}=\left[\begin{array}{l}B_{1} \\ B_{2}\end{array}\right], \quad H_{k}=\left[\begin{array}{ll}I_{4} & \emptyset_{4}\end{array}\right]$,

where $A_{1}=\Delta t \cdot I_{4}, B_{1}=\left(\Delta t^{2} / 2\right) \cdot I_{4}, B_{2}=\Delta t \cdot I_{4}, I_{4}$ is the $4 \mathrm{x} 4$ identity matrix, $\emptyset_{4}$ is the $4 \times 4$ zero matrix and $\Delta t$ is the time interval between the consecutive scanning frames of the laser. The state transition matrix $A$ encodes the kinematics of the legs, the input matrix the acceleration effects in the position and the velocity of the legs, while the observation matrix encodes the fact that the only observables are the position of the legs. As noted before, we have no knowledge of the input (of the acceleration). Hence, we model the acceleration as a random variable, following a zero mean normal distribution. The normal distribution of acceleration $p\left(a_{k}\right) \sim N\left(0, C_{k}\right)$ was experimentally defined, and describes the acceleration uncertainty throughout the gait. Its covariance matrix $C_{k}$ is a $4 \times 4$ diagonal matrix with diagonal elements $\sigma_{a_{x}^{L}}^{2}, \sigma_{a_{y}^{L}}^{2}$ $\sigma_{a_{x}^{R}}^{2}, \sigma_{a_{y}^{R}}^{2}$, which are the variances of the legs' accelerations 
along the axes. The acceleration influences the model as noise. Since we are using the information for the variability of the system's input $C_{k}$ to define the process noise, the matrix $Q_{k}$ is computed by the relation: $Q_{k}=B_{k} \cdot C_{k} \cdot B_{k}{ }^{T}$. The standard deviations of the measurement noise $v_{k}$ along the axes are $v_{x_{k}}^{2}$ and $v_{y_{k}}^{2}$ for both legs, which are the diagonal elements of the $4 \times 4$ diagonal matrix $R_{k}$.

The predictions from the KF are used as seed for the Kmeans++ algorithm, [35]. Around the predicted positions of the legs, leg-windows are set, having initial constant dimensions proportional to the leg-circle's dimensions. However, the leg-windows dimensions are also adaptively adjusted, by enlarging or shortening them according to the variability of the KF estimates. From the two leg-windows, a wider search window is defined in the plane, and the detected raw data inside it, are ready to be preprocessed. Thus, the described process results in an iterative interaction between detection and tracking processes.

\section{False Detection Treatment}

False detections are the cases in which either one leg is occluded by the other or there is interference of another person's legs inside the search window that have not been successfully discarded, Fig. 3. Those cases can interrupt or contaminate the detection and can result in losing track of the legs. To address such false detections, certain hypotheses are checked. If the detected leg centers violate a Euclidean distance constraint that we have set, relevant to the mean pelvic width, or when there are detected less or more than two laser groups, the corresponding detection is regarded false. In order to continue with the tracking phase, an onlyprediction Kalman filter is applied. In that particular case, we perform only the prediction step and, following the usual literature method, we use the prediction state vector $\hat{x}_{k \mid k-1}$ and the a priori estimate covariance $P_{k \mid k-1}$ as the estimates fed to the next step, disregarding the observations for time frame $k$.

\section{EXPERIMENTAL ANALYSIS \& EVALUATION}

\section{A. Experiment and data description}

The experimental data used in this paper were collected using a HOKUYO rapid laser sensor (UBG-04LX-F01, with mean sampling period of about $28 \mathrm{~ms}$ ) mounted on the robotic rollator of Fig. 1. For the evaluation of our algorithmic procedure, we have used the recorded data of six patients (over 65 years old) performing a scenario during which the user, with physical support of the rollator, walks straight with maximum and constant velocity over a walkway. All participants are persons with mild motion impairment, that they were wearing their normal clothes (no need of specific clothing). The experimental procedure has been performed in a Geriatric Hospital, under appropriate carer's supervision. The length of the path differs depending on the subjects ability to walk. The subjects were instructed to walk as normally as possible. This results in a different walking speed for each subject, and in a different step length. A sequence of video snapshots of the experimental recordings procedure during a full stride ${ }^{1}$ execution from subjects are presented in Fig. 4. The motion caption markers in those snapshots are not used in the current framework for simplicity.

\section{B. Evaluation Results: Detection and Tracking}

In this section we have evaluated the successful performance of our system for the detection and tracking of patients' legs, which is a perquisite for modelling the gait cycle phases. All the noise parameters for Kalman Filter tuning and the other constants and thresholds of the detection and tracking system have been experimentally computed for the particular patient-dataset. In Fig. 5, six snapshots from one stride along with the detected user's legs are depicted. The subfigures correspond to the gait phases: (a) LR, (b) MS, (c) TS, (d) PW, (e) IW, (f) MW, accordingly. Each figure shows a wide planar area in front of the laser scanner. The raw laser data are depicted with blue stars, presenting both the leg clusters and outliers, while the magenta circles are the fitted circles of the legs clusters. The black rectangle enclosing the legs circles represents the search window for each scanning frame. Finally, the detected leg centers are depicted with black x's and their estimated positions with green $x$ 's.

For the detection process, our algorithmic approach, for the six patients that performed the described scenario, resulted in $100 \%$ successful detection for four patients, while the other two patients had $94.2 \%$ and $96.9 \%$ success. In those two cases we noticed false detection of the user's legs, due to occlusion while turning or due to the proximal position of the carer's legs which are included in the search window. While we lose detection, the tracking of the patient's legs is not lost since we are using the False detection treatment routine, which was described previously.

For the evaluation of how much KF estimated positions improve the detected leg centers, we computed the Root Mean Square Errors (RMSE). The average RMSE for each leg, computed by comparing the detected and estimated positions were $1.96 \mathrm{~mm}$ for the $\mathrm{x}$ coordinate (lateral motion) and $1.4 \mathrm{~mm}$ for the y coordinate (forward motion). In the lateral plane, there is greater uncertainty due to the leg clusters shape deformability and length variability. In the absence of ground truth, we cannot evaluate accurately these errors. However, the smoothing effect of KF can be ascertained by Fig. 6, which presents an example of the detected and estimated leg positions for the lateral and forward motion with respect to the scanning frame for one patient. The green and magenta lines represent the detected leg centers' displacement, while the red and blue stars their estimated displacement along the axes for the right and left leg accordingly. The depicted results confirm the filtering efficiency of the KF on the detected leg centers, by removing noisy peaks, especially in the lateral displacement caused by the laser cluster's shape and length variability, while walking with respect to the laser scanner.

\footnotetext{
${ }^{1}$ Stride is the equivalent of gait cycle, i.e. two sequential steps define one stride, [33].
} 


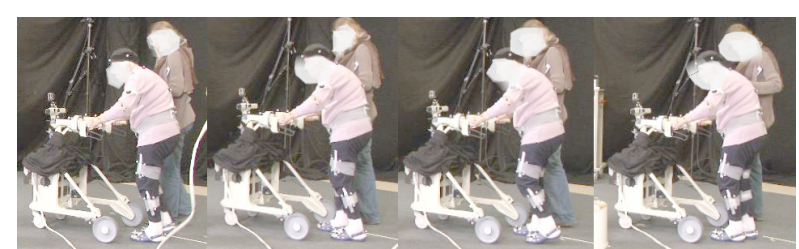

Fig. 4: A sequence of snapshots during a full stride execution from a female subject. From left to right images: Loading Response, Terminal Stance, Pre-swing, and Terminal Swing.

\section{Evaluation Results: Gait recognition}

HMM training procedure comprises only a part of subjects' data, excluding the recorded data of one subject. The testing procedure aims to test the performance of the proposed approach, validating its generalisation capacity over unseen data obtained by new subjects. The evaluation is based on an assessment of the estimated states provided by the constructed HMM, which represents the human gait cycle. The experimental results were done by using only one Gaussian mixture (for simplicity).

For testing and evaluation purposes of the constructed HMM, we have demonstrated an example of the real experimental data set which is depicted in Fig. 7a. The goal of this evaluation phase is to unveil the hidden parts of the constructed models, i.e. to estimate the correct sequence of phase transitions that occur in the test data. This test dataset reflects the gait session of one elderly subject, and comprises about eleven walking sections (about eleven strides ${ }^{1}$ ). In this figure the displacement of each leg in the sagittal plane with respect to time is depicted on the top graph, while the bottom graph shows the evolution of the distance between legs within the same time frame. This figure is very useful to understand the exact subject's motion. The walking session is starting with the left leg, and it is obvious from the increasing of the distance between the legs that the early gait phases are occurred, Fig. 7a. While this distance is going to zero (crossing point) the right leg is moving forward until the next crossing point. It can be observed that the first stride is completed close to $2 \mathrm{sec}$. This is observable to the results of the constructed HMM in Fig. $7 \mathrm{~b}$, since at the time instant just before $2 s e c$, a gait cycle is completed by the recognized MW phase.

The estimated sequence of gait phases obtained using the trained model is depicted in Fig. 7b. This figure shows the time instant at which each gait phase (hidden state of HMM) is activated. A first remark that can be made by observing these experimental results is that the evolution of the gait phases provided by the models matches the general evolution of the human gait model that is to be represented by the HMMs; i.e. the gait phases appear sequentially with the correct order, and the time frame of each phase is within the general bounds as have been mentioned previously in Section II. It is obvious that some of the gait phases are omitted, since these experimental data corresponds to a subject with walking difficulties related to an underlying pathology. Some of the gait phases seem to be difficult to be recognized for the specific experimental data set (patient). Also, it is known,

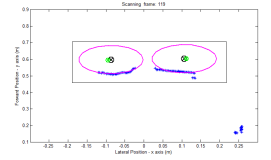

(a) LR

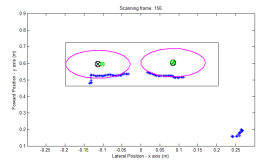

(d) PW

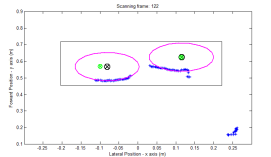

(b) MS

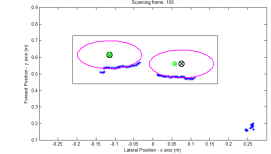

(e) IW

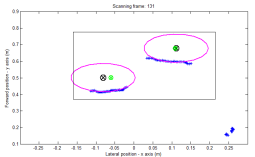

(c) TS

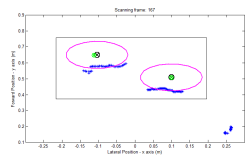

(f) $\mathrm{MW}$
Fig. 5: Snapshots from a stride with respect to the laser, showing the results of the detection and tracking process
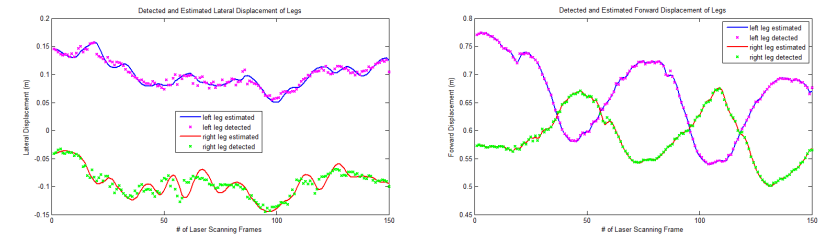

(a) Detected and estimated legs (b) Detected and estimated legs lateral displacement

forward displacement

Fig. 6: Example of detected and estimated lateral and forward legs' displacement w.r.t. the laser scanning frame
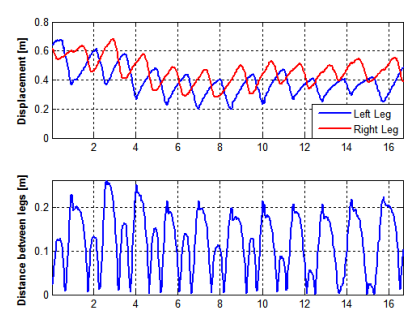

(a)

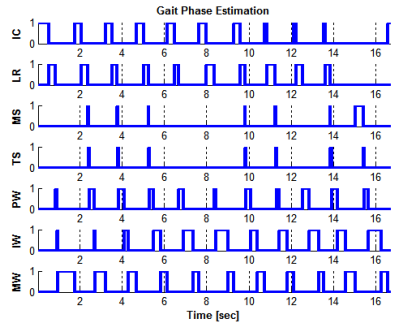

(b)
Fig. 7: (a) Real experimental data from one subject's gait that have been used in the testing and evaluation phase of constructed HMM. Top: Left (blue data) and Right (red data) legs displacement. Bottom: legs distance in the sagittal plane. (b) Estimated sequence of gait states based on the constructed model with respect to time by testing the data depicted in left figure which represent an unknown walking section.

that specific pathologies may affect the walking activity in an indistinct way, that reflects accidental the gait performance and the appearance of gait phases.

There is an assumption, without loss of generality, that at the beginning of each gait cycle the initial contact refers to the left leg, while a complete stride is concluded when the right leg is again in front of the left leg, ready for a new initial contact and therefore for the next stride. By observing the results depicted in Fig. 7b, it can be seen that the model manages to successfully recognize that (for the recorded experimental data of Fig. 7a, used in this case study for model testing) the subject starts the motion with the left leg. Thus, the first estimated gait phase in Fig. 7b is Initial Contact (IC).

Another remark concerns the terminal instant of the motion. At the end of the recorded test data of Fig. 7a 
(after $16 \mathrm{sec}$ ), it can be seen that the motion is terminated approximately at the beginning of a stride (start a new stride with the left leg). Indeed, as shown in Fig. 7b, the model correctly recognizes that the motion corresponding to the test data of Fig. 7a is terminated (after 16sec) in a phase at the beginning of a gait cycle (IC in Fig. 7b). The results show that the constructed model recognizes the pathological gait.

\section{CONCLUSIONS AND FUTURE WORK}

In this paper, a framework to represent and analyse human pathological gait using a Hidden Markov Model (HMM) is presented. The HMM employed in this work analyses gait phases by using the features estimated by a forward backward system of detection and tracking of the patients' legs from raw data provided by a typical laser rangefinder sensor, thus constituting a completely noninvasive approach using a non-wearable device. The proposed pathological gait modeling method has been applied to actual patient gait data. The successful estimation of the gait parameters fed the gait analysis system. The experimental results clearly show that this method is capable of correctly recognizing human pathological gait cycle patterns. Furthermore, this approach, based on its statistical learning properties, is quite flexible and readily extensible to different gait models, thus presenting a strong potential to support a behaviour-based cognitive robot control framework.

For further research, we will investigate the potential to apply this methodology to the classification problem between normal and pathological walking pattern. Therefore, a variety of abnormal gaits (corresponding to specific motor impairments) can be characterized by different models. Furthermore, within our future plans is to model more gait patterns based on HMM, regarding turning motions during indoor ambulation, as well as more complicated and maneuvering motions that appear in daily activities. Towards this end we are working on a new detection and tracking system based on particle filtering. The aim is to create a system that can detect in real time specific gait pathologies and automatically classify the patient status or the rehabilitation progress, thus providing the necessary information for effective cognitive (context-aware) active mobility assistance robots.

\section{REFERENCES}

[1] P. D. Foundation, "Statistics for parkinson's disease," 2010.

[2] S. Center, "Stroke statistics," 2010.

[3] USCensus, "The elderly population," 2010.

[4] X. et. al., "Advances in intelligent mobility assistance robot integrating multimodal sensory processing," Lecture Notes in Computer Science, Universal Access in Human-Computer Interaction. Aging and Assistive Environments, vol. 8515, pp. 692-703, 2014.

[5] P. Brezillon, "Context in problem solving: A survey," The Knowledge Engineering Review, vol. 14, pp. 1-34, 1999.

[6] S. D. et.al., "Pamm - a robotic aid to the elderly for mobility assistance and monitoring: A 'helping-hand' for the elderly," in IEEE Int'l Conf. on Robotics and Automation, 2000, pp. 570-576.

[7] M. Spenko, H. Yu, and S. Dubowsky, "Robotic personal aids for mobility and monitoring for the elderly," IEEE Trans. on Neural Systems and Rehabilitation Engineering, 2006.

[8] S. Jiang, B. Zhang, and D. Wei, "The elderly fall risk assessment and prediction based on gait analysis," in IEEE 11th Int'l Conf. on Computer and Information Technology, 2011, pp. 176-180.
[9] M. Bachlin, M. Plotnik, D. Roggen, I. Maidan, J. Hausdorff, N. Giladi, and G. Troster, "Wearable assistant for parkinson's disease patients with the freezing of gait symptom," IEEE Trans. on Information Technology in Biomedicine, vol. 14, no. 2, pp. 436-446, 2010.

[10] J. F.-S. Lin and D. Kulic, "Automatic human motion segmentation and identification using feature guided $\mathrm{hmm}$ for physical rehabilitation exercises," in IEEE Int'l Conf. on Intell. Robots \& Syst., 2011.

[11] J. Bae and M. Tomizuka, "Gait phase analysis based on a hidden markov model," Mechatronics, vol. 21, no. 6, pp. 961 - 970, 2011.

[12] C. Nickel, C. Busch, S. Rangarajan, and M. Mobius, "Using hidden markov models for accelerometer-based biometric gait recognition," in 2011 IEEE 7th Int'l Colloquium on Signal Process. and its Applic.

[13] I. Pappas, M. Popovic, T. Keller, V. Dietz, and M. Morari, "A reliable gait phase detection system," IEEE Trans. on Neural Systems and Rehabilitation Engineering, vol. 9, no. 2, pp. 113-125, 2001.

[14] G. e. Bebis, "An architecture for understanding intent using a novel hidden markov formulation," Int'l J. of Humanoid Robotics, 2008.

[15] M. Meng, Q. She, Y. Gao, and Z. Luo, "Emg signals based gait phases recognition using hidden markov models," in Information and Automation (ICIA), 2010 IEEE Int'l Conf. on, 2010, pp. 852-856.

[16] A. Panangadan, M. Mataric, and G. Sukhatme, "Tracking and modeling of human activity using laser rangefinders," Int'l Journal of Social Robotics, vol. 2, no. 1, pp. 95-107, 2010.

[17] X. Shao, H. Zhao, K. Nakamura, R. Shibasaki, R. Zhang, and Z. Liu, "Analyzing pedestrians' walking patterns using single-row laser range scanners," in IEEE Int'l Conf. on Systems, Man and Cybernetics, 2006.

[18] T. Horiuchi, S. Thompson, S. Kagami, and Y. Ehara, "Pedestrian tracking from a mobile robot using a laser range finder." IEEE Int'l Conf. Systems, Man and Cybernetics, ISIC, October 7-10 2007.

[19] K.Arras and et.al., "Efficient people tracking in laser range data using a multi-hypothesis leg-tracker with adaptive occlusion probabilities." IEEE Int'l Conf. on Robotics \& Automation, 2008.

[20] G.Lee, E.Jung, T.Ohnuma, N.Chong, and B.Yi, "Jaist robotic walker control based on a two-layered kalman filter." Shangai, China: 2011 IEEE Int'l Conf. on Robotics and Automation, May 2011.

[21] T.Ohnuma, G.Lee, and N.Chong, "Particle filter based feedback control of jaist active robotic walker." Atlanta, Georgia: 2011 ROMAN, 20th IEEE Int'1 Symp. on Robot and Human Interactive Communication, 31 July - 3 August 2011.

[22] H. Kim, W. Chung, and Y. Yoo, "Detection and tracking of human legs for a mobile service robot," in IEEE/ASME Int'l Conf. onAdvanced Intelligent Mechatronics (AIM), 2010, 2010, pp. 812-817.

[23] W.-H. Mou, M.-F. Chang, C.-K. Liao, Y.-H. Hsu, S.-H. Tseng, and L.-C. Fu, "Context-aware assisted interactive robotic walker for parkinson's disease patients," in IEEE/RSJ Int'l Conf. on Intelligent Robots and Systems (IROS), 2012, Oct 2012, pp. 329-334.

[24] L. R. Rabiner, "Readings in speech recognition," A. Waibel and K.-F. Lee, Eds. San Francisco, CA, USA: Morgan Kaufmann Publishers Inc., 1990, ch. A tutorial on hidden Markov models and selected applications in speech recognition, pp. 267-296.

[25] A. Katsamanis, G. Papandreou, and P. Maragos, "Face active appearance modeling and speech acoustic information to recover articulation," IEEE Trans on Audio, Speech, and Language Processing, 2009.

[26] S. Theodorakis, A. Katsamanis, and P. Maragos, "Product-HMMs for automatic sign language recognition." in ICASSP, 2009.

[27] P. Turaga, R. Chellappa, V. S. Subrahmanian, and O. Udrea, "Machine recognition of human activities: A survey," Circuits and Systems for Video Technology, IEEE Trans. on, vol. 18, no. 11, 2008.

[28] C. Chen, J. Liang, H. Zhao, and H. Hu, "Gait recognition using hidden markov model," in Advances in Natural Computation.

[29] A. Kale, A. N. Rajagopalan, N. Cuntoor, and V. Kruger, "Gait-based recognition of humans using continuous hmms," in Proc. 5th IEEE Int'l Conf. onAutomatic Face and Gesture Recognition, 2002.

[30] A. Mannini and A. M. Sabatini, "Accelerometry-based classification of human activities using markov modeling," p. 10, 2011.

[31] X. Papageorgiou, G. Chalvatzaki, C. Tzafestas, and P. Maragos, "Hidden markov modeling of human normal gait using laser range finder for a mobility assistance robot," in Proc. of the 2014 IEEE Int'l Conf. on Robotics and Automation.

[32] R.-H. T. F. Inman, V.T., Human walking. Williams \& Wilkins, 1981.

[33] Gait Analysis: Normal and Pathological Function.

[34] A primer of orthopaedic biomechanics.

[35] D. Arthur and S. Vassilvitskii, "K-means++: The advantages of careful seeding," in Proc. of the 18th Annual ACM-SIAM Symposium on Discrete Algorithms, ser. SODA '07, 2007. 